\title{
Comparison of culture media for the laboratory diagnosis of chancroid
}

\author{
A. PILLAY, A. A. HOOSEN*, D. LOYKISSOONLAL, C. GLOCK, B. ODHAV and A. W. STURM \\ Department of Medical Microbiology and MRC Genital Ulcer Disease Research Unit, Faculty of Medicine, \\ University of Natal, Durban, South Africa
}

\begin{abstract}
Seven different agar-based media were compared to determine the optimal set of culture media for primary isolation of Haemophilus ducreyi. Also, a new method for sampling genital ulcers - with a disposable sterile plastic loop - and processing specimens that provides a standardised inoculum for culture of $\boldsymbol{H}$. ducreyi on various media is described. A total of 202 patients with genital ulcer disease was enrolled in this study. A sterile swab or plastic loop was used to sample the base of the ulcers and ulcer material was suspended in sterile phosphate-buffered saline. A 100- $\mu$ l sample of this suspension was mixed with an equal volume of tryptic soy broth containing IsoVitaleX and centrifuged for $1 \mathrm{~min}$. This suspension was used to inoculate the different media. Plates were incubated at $33^{\circ} \mathrm{C}$ in micro-aerophilic conditions and examined for growth of $H$. ducreyi after $48 \mathrm{~h}$. Of the 202 specimens, $77(38.1 \%)$ were culture positive for $H$. ducreyi. None of the agar bases supported the growth of all $\boldsymbol{H}$. ducreyi strains. Based on this observation, we recommend the universal use of Mueller-Hinton agar base supplemented with chocolate horse blood and IsovitaleX (MH-HBC) and Columbia agar base supplemented with bovine haemoglobin, activated charcoal, fetal calf serum and IsovitaleX ( $\mathrm{C}-\mathrm{HgCh})$ to enable comparison of prevalence figures for chancroid. In addition, the novel sampling technique described in this study eliminates sampling bias normally associated with genital ulcer specimens.
\end{abstract}

\section{Introduction}

Chancroid, caused by Haemophilus ducreyi, is endemic in many developing countries including South Africa [1-3]. The interaction of chancroid and human immunodeficiency virus (HIV) is of major concern as genital ulcers have been shown to increase the risk of HIV transmission $[4,5]$. With the rapidly increasing numbers of individuals with genital ulcers that are being co-infected with HIV, it is of paramount importance to improve the specificity and sensitivity of diagnostic tests for the genital ulcer diseases (GUDs), in particular chancroid. Recently, Orle et al. [6] reported a multiplex PCR assay for the diagnosis of syphilis, chancroid and HSV types 1 and 2 infection. This seems promising and will result in a rapid,

Received 29 July 1997; revised version accepted 13 Feb. 1998.

Corresponding author: Professor A. W. Sturm.

*Present address: Department of Medical Microbiology, PO Box 211, Medunsa 0204, South Africa. sensitive method for the diagnosis of chancroid compared with culture. However, presently, culture of $H$. ducreyi remains the 'gold standard' for the laboratory diagnosis of chancroid. Furthermore, culture is essential to monitor antimicrobial susceptibility patterns. For primary isolation, it is well known that all strains of $H$. ducreyi do not grow on any one particular medium and it has been suggested that this strain variation is due to nutritional requirements [7-9]. The sensitivity of culture techniques for detecting $H$. ducreyi have been reported to vary from $2 \%$ [10] to $84 \%$ [9]. In most studies inoculation of plates is done with swabs used for collection of the specimen from the ulcer. As a result, the yield on different plates is influenced by the sequence of inoculation. If studies comparing growth media are nested in a broader study on the aetiology of genital ulcers, then an additional confounding factor will be the sequence in which swabs for the different investigations are taken. The present study was undertaken to assess the optimal set of culture media required for primary isolation and remove bias related to the collection of consecutive specimens and subsequent processing in the laboratory, using a novel collection technique for genital ulcer specimens. 


\section{Materials and methods}

A total of 202 patients with GUD, presenting to the City Health STD Clinic in Durban, was enrolled in this study. Before the specimens were collected, the surfaces of the ulcers were wiped with sterile gauze saturated in sterile phosphate-buffered saline (PBS), $\mathrm{pH}$ 7.3. For the first 58 patients, sterile cotton swabs (Benmore Diagnostics, South Africa) were used to collect material from the base of the ulcers. To release ulcer material, swabs were rolled and pressed simultaneously against the side of a sterile cryotube (Corning, Canada) containing $1 \mathrm{ml}$ of sterile PBS. For the remaining 144 patients, a disposable sterile plastic 10- $\mu 1$ loop (Copan, Italy) was used to scrape the cleaned base of the ulcer and the exudate was suspended in $1 \mathrm{ml}$ of PBS. The PBS suspension was vortex mixed for $3 \mathrm{~s}$ and an equal volume of Tryptic Soy Broth (Difco) containing IsoVitaleX (Becton Dickinson, Cockeysville, USA) $1 \%$ was added to $100 \mu \mathrm{l}$ of ulcer specimen suspension. An average of 12 ulcer specimens was collected per day and transported, in two batches, to the laboratory for further processing, thus ensuring that specimens were processed within $2.5 \mathrm{~h}$ of sampling.
On arrival in the laboratory, the specimens were vortex mixed for another $3 \mathrm{~s}$ and centrifuged at $13500 \mathrm{~g}$ for $1 \mathrm{~min}$. $60 \mu \mathrm{l}$ of the supernate were discarded and the sediment was resuspended in the remaining $140 \mu 1$. Then, $20-\mu 1$ volumes were dispensed on to the agar surface of seven different media (Table 1). The inoculum was streaked across the agar surface of the different plates which were then incubated at $33^{\circ} \mathrm{C}$ under micro-aerophilic conditions and examined for growth of $H$. ducreyi after $48 \mathrm{~h}$. Suspected colonies were identified as $H$. ducreyi as described previously [16].

\section{Results}

Of the 202 specimens 77 (38.1\%) yielded $H$. ducreyi on culture. Of the specimens collected with the cotton swabs, $21(36 \%)$ of 58 yielded $H$. ducreyi whereas 56 $(39 \%)$ of 149 collected with the plastic loop yielded this organism. Specimens collected with the loop yielded more $H$. ducreyi colonies.

Table 2 shows the yield of $H$. ducreyi on individual culture media. There was not a single agar base

Table 1. Composition of media for primary isolation of $H$. ducreyi

\begin{tabular}{llc}
\hline Medium & Composition & Reference \\
\hline C-HgCh & C, bovine haemoglobin (Difco) $1 \%$, activated charcoal $0.2 \%$, FCS $5 \%$, IsoVitaleX $1 \%$, vancomycin $3 \mu \mathrm{g} / \mathrm{ml}$ & $*$ \\
C-HB & C, IsoVitaleX 2\%, horse blood $16.5 \%$, vancomycin $3 \mu \mathrm{g} / \mathrm{ml}$ & {$[11]$} \\
GC-CtS & GC, FBS $10 \%$, IsoVitaleX $1 \%$, catalase $200 \mu \mathrm{g} / \mathrm{ml}$, vancomycin $3 \mu \mathrm{g} / \mathrm{ml}$ & {$[12]$} \\
GC-FHB & GC, Filde's extract (Oxoid) $5 \%$, horse blood $5 \%$, vancomycin $3 \mu \mathrm{g} / \mathrm{ml}$ & {$[9]$} \\
GC-HgS & GC, bovine haemoglobin $1 \%$, FCS $5 \%$, IsoVitaleX $1 \%$ & {$[13]$} \\
MH-Ch & MH, bovine haemoglobin $1 \%$, activated charcoal $0.2 \%$, IsoVitaleX $1 \%$, vancomycin $3 \mu \mathrm{g} / \mathrm{ml}$ & {$[14]$} \\
MH-HBC & MH, chocolate horse blood $5 \%$, IsoVitaleX $1 \%$, vancomycin $3 \mu \mathrm{g} / \mathrm{ml}$ & {$[15]$} \\
\hline
\end{tabular}

C, Columbia agar base (Difco); FCS, fetal calf serum (Whittaker); GC, Gonococcal agar base (Gibco); FBS, fetal bovine serum (Whittaker); MH, Mueller-Hinton agar base (Becton Dickinson).

* Dangor and Ballard, personal communication.

Table 2. Primary isolation of $H$. ducreyi on different media

\begin{tabular}{lccc}
\hline $\begin{array}{l}\text { Media or combination } \\
\text { of medium }\end{array}$ & $\begin{array}{c}\text { Number }(\%) \text { of all } \\
\text { isolates }(\mathrm{n}=77)\end{array}$ & $\begin{array}{c}\text { Positive patients } \\
(\mathrm{n}=202)\end{array}$ & $\begin{array}{c}\text { Isolates on this } \\
\text { medium alone }\end{array}$ \\
\hline C-HgCh & $57(74.0)$ & 28.2 & 2 \\
GC-CtS & $52(67.5)$ & 25.7 & 1 \\
MH-HBC & $50(64.9)$ & 24.7 & 1 \\
GC-HgS & $49(63.6)$ & 24.2 & 0 \\
MH-Ch & $49(63.6)$ & 24.2 & 1 \\
GC-FHB & $36(46.7)$ & 17.8 & 0 \\
C-HB & $31(40.2)$ & 15.3 & 0 \\
MH-HBC + C-HgCh & $74(96.1)$ & 36.6 & 0 \\
C-HgCh + GC-CtS & $71(92.2)$ & 35.1 & 0 \\
MH-HBC + GC-HgS & $70(90.9)$ & 34.6 & 0 \\
MH-Ch + GC-HgS & $69(89.6)$ & 34.1 & 0 \\
MH-Ch + C-HgCh & $68(88.3)$ & 33.6 & 0 \\
MH-HBC + GC-CtS & $67(87.0)$ & 33.1 & 0 \\
GC-HgS + C-HgCh & $65(84.4)$ & 32.1 & 0 \\
GC-FHB + C-HgCh & $65(84.4)$ & 32.1 & 0 \\
MH-Ch + GC-CtS & $65(84.4)$ & 32.1 & 0 \\
C-HgCh + C-HB & $64(83.1)$ & 31.6 & 0 \\
GC-HgS + C-HB & $63(81.8)$ & 31.1 & 0 \\
GC-HgS + GC-CtS & $62(80.5)$ & 30.6 & 0 \\
GC-CtS + C-HB & $61(79.2)$ & 30.1 & 0 \\
MH-HBC + MH-Ch & $58(75.3)$ & 28.7 & 0 \\
MH-HBC + GC-FHB & $58(75.3)$ & 28.7 & 0 \\
GC-FHB + MH-Ch & $57(74.0)$ & 28.2 & \\
GC-FHB + GC-HgS & $57(74.0)$ & 28.2 & \\
\hline
\end{tabular}


identified that supported the growth of all isolates. The Columbia agar base supplemented with haemoglobin, IsoVitaleX and fetal calf serum and with activated charcoal (C-HgCh) showed the best results, i.e., 57 $(74 \%)$ of 77 isolates were grown on this medium. However, it took a combination of four media to support the growth of all isolates. This set of four included all three agar bases, i.e., Columbia, Gonococcal and Mueller-Hinton; the only common component in all the media formulations was IsoVitaleX.

In view of the fact that most laboratories use a set of two culture media for the laboratory diagnosis of $H$. ducreyi infection, the yield on every possible combination of two was calculated. The results are shown in Table 2. The combination of $\mathrm{MH}-\mathrm{HBC}$ and $\mathrm{C}-\mathrm{HgCh}$ supported the growth of $74(96 \%)$ of the 77 isolates. Of the three isolates that were not cultured on the $\mathrm{MH}-\mathrm{HBC}$ and $\mathrm{C}-\mathrm{HgCh}$ pair, one isolate was grown on GC-CtS, GC-HgS and C-HB, another on $\mathrm{MH}-\mathrm{Ch}$ medium alone and the third on GC-CtS medium alone.

\section{Discussion}

Studies of the aetiology of GUD have serious limitations because of the fact that specimen requirements differ for the diagnosis of the different causative agents. The usual practice is to take several consecutive swabs which are then placed in the respective transport media randomly or on the basis of clinical probability. This practice has two major disadvantages: the ratio between the amount of representative material to debris with colonisers will be different on the respective swabs and patients with painful ulcers will not allow appropriate samples to be taken very frequently. In view of these factors, the quality of these consecutive specimens will differ. Attempts to rectify this can be made by random distribution in the different transport media; however, large numbers of patients are needed to eliminate sampling bias.

A similar problem arises when assessing efficacy of culture: if large numbers of media have to be inoculated with one swab, loss of specimen from the swab during this process would invalidate the observation unless the sequence of inoculation is randomised and the numbers of positive cultures are very large. In this study, the technique of specimen collection was standardised to eliminate bias in determining the aetiology of genital ulcers and to enable the evaluation of culture media for the isolation of $H$. ducreyi. The ulcer material was suspended in PBS to allow for distribution over different transport media. For culture of $H$. ducreyi, the PBS suspension was added to tryptic soy broth to maintain viability of the organisms during transport. An equal volume of this suspension was used to inoculate all culture media.
After the first 58 patients, the sampling method was changed from a cotton swab to a plastic loop. This change was made because the type of swab which gives optimal yield for different GUD organisms varies and this study formed part of another study on the aetiology of GUD (unpublished observations) which would be invalidated by the use of swabs. Although the present study was not designed to compare the two sampling methods, growth of $H$. ducreyi was slightly better when the loop was used for sampling, suggesting the presence of inhibitory material in the swab or absorption of organisms in the cotton wool. In general, the number of colonies obtained when the loop was used for sampling was much larger than when a swab was used.

Although several media formulations have been reported for the primary isolation of $H$. ducreyi, the sensitivities of these media differ considerably. In the present study, Columbia agar base containing bovine haemoglobin, activated charcoal and fetal calf serum supplemented with IsoVitaleX $(\mathrm{C}-\mathrm{HgCh})$ gave the highest sensitivity with $74 \%$ of isolates being cultured. In contrast, Columbia agar base containing lysed horse blood supplemented with IsoVitaleX (C-HB) (modified Bieling medium), supported the growth of only $40 \%$ of isolates. This medium has been reported to give a high yield compared with the combination of $\mathrm{MH}$ HBC and GC-HgS [12]. Its performance is supposed to be highly dependent on the water binding capacity of the agar with a 'sloppy' agar giving better results than a firm one [11]. Alternatively, the lower isolation rate may be due to the use of the agar base from different suppliers (Oxoid versus Difco) or the use of IsoVitaleX instead of yeast dialysate. The modified Bieling medium with yeast dialysate [11] is used routinely in this laboratory for culturing laboratory strains of $H$. ducreyi and it has been observed that strains grow better when this medium is supplemented with yeast dialysate rather than IsoVitaleX (unpublished observations). However, the production of yeast dialysate is cumbersome and needs strict quality control $[11,17]$.

When the single best medium (C-HgCh) was used, $26 \%$ of isolates were not cultured. This further supports the finding of other workers that optimal isolation rates for $H$. ducreyi cannot be achieved with a single medium $[9,15]$.

Nsanze et al. [15] recommended a combination of two media (MH-HBC and GC-HgS) for the primary isolation of $H$. ducreyi. Subsequently, Dangor et al. [9] also showed that optimal isolation rates were achieved with this combination. In the present study, the combination of $\mathrm{MH}-\mathrm{HBC}$ and $\mathrm{C}-\mathrm{HgCh}$ was more sensitive for the culture of $H$. ducreyi than $\mathrm{MH}-\mathrm{HBC}$ and $\mathrm{GC}-\mathrm{HgS}$ (74\% versus $70 \%$ respectively), although the difference is not significant statistically $(\mathrm{p}>0.5$, $\chi^{2}$ test). However, a combination of four media (i.e., 
MH-HBC, GC-HgS, C-HgCh, GC-CtS) was needed to grow all 77 isolates. The use of four different media for the cultural diagnosis of chancroid is labour intensive, time-consuming and difficult to implement - especially in circumstances with poor resources.

Five strains were able to grow on only one medium (Table 2), corroborating the suggestion that there is variation among strains with respect to nutritional requirements. Also, in support of this is the finding that none of the agar bases supported the growth of all $H$. ducreyi strains. At present, there is no consensus on the type or combination of media to use for routine primary isolation of $H$. ducreyi. As long as there is no single medium or combination of two that optimally supports growth of all $H$. ducreyi strains, we recommend the universal use of the $\mathrm{MH}$ $\mathrm{HBC}$ and $\mathrm{C}-\mathrm{HgCh}$ combination to allow comparison of prevalence figures for chancroid.

\section{References}

1. Coovadia YM, Kharsany A, Hoosen A. The microbial aetiology of genital ulcers in black men in Durban, South Africa. Genitourin Med 1985; 61: 266-269.

2. Plummer FA, D' Costa LJ, Nsanze H et al. Clinical and microbiologic studies of genital ulcers in Kenyan women. Sex Transm Dis 1985; 12: 193-197.

3. Meheus A, Van Dyck E, Ursi JP, Ballard RC, Piot P. Etiology of genital ulcerations in Swaziland. Sex Transm Dis 1983; 10: 33-35.

4. Telzak EE, Chiasson MA, Bevier PJ, Stoneburner RL, Castro $\mathrm{KG}$, Jaffe HW. HIV-1 seroconversion in patients with and without genital ulcer disease. A prospective study. Ann Intern Med 1993; 119: 1181-1186.

5. Behets FM-T, Liomba G, Lule G et al. Sexually transmitted diseases and human immunodeficiency virus control in Malawi: a field study of genital ulcer disease. $J$ Infect Dis 1995; 171: 451-455.

6. Orle KA, Gates CA, Martin DH, Body BA, Weiss JB. Simultaneous PCR detection of Haemophilus ducreyi, Treponema pallidum, and Herpes simplex virus types 1 and 2 from genital ulcers. J Clin Microbiol 1996; 34: 49-54.

7. Dylewski J, Nsanze H, Maitha G, Ronald A. Laboratory diagnosis of Haemophilus ducreyi: sensitivity of culture media. Diagn Microbiol Infect Dis 1986; 4: 241-245.

8. Macdonald $\mathrm{K}$, Cameron DW, Irungu $\mathrm{G}$ et al. Comparison of Sheffield media with standard media for the isolation of Haemophilus ducreyi. Sex Transm Dis 1989; 16: 88-90.

9. Dangor Y, Miller SD, Koornhof HJ, Ballard RC. A simple medium for the primary isolation of Haemophilus ducreyi. Eur J Clin Microbiol Infect Dis 1992; 11: 930-934.

10. Chapel TA, Brown WJ, Jeffres C, Stewart JA. How reliable is the morphological diagnosis of penile ulcerations? Sex Transm Dis 1977; 4: 150-152.

11. Sturm AW. Clinical and bacteriological aspects of ulcus molle. $\mathrm{PhD}$ thesis, University of Amsterdam, 1981.

12. Totten PA, Stamm WE. Clear broth and plate media for culture of Haemophilus ducreyi. J Clin Microbiol 1994; 32: 20192023.

13. Duncan MO, Bilgeri YR, Fehler HG, Ballard RC. The diagnosis of sexually acquired genital ulcerations in black patients in Johannesburg. S Afr Med J 1981; 1: 20-23.

14. Lockett AE, Dance DAB, Mabey DCW, Drasar BS. Serum-free media for isolation of Haemophilus ducrevi. Lancet 1991; 338: 326.

15. Nsanze H, Plummer FA, Maggwa $\mathrm{ABN}$ et al. Comparison of media for the primary isolation of Haemophilus ducreyi. Sex Transm Dis 1984; 11: 6-9.

16. Sturm AW, Zanen HC. Characteristics of Haemophilus ducreyi in culture. J Clin Microbiol 1984; 19: 672-674.

17. Kunimoto DY, Slaney L, Koss J et al. Field testing of modified Bieling media for the isolation of Hatmophilus ducreyi in Kenya. Eur J Clin Microbiol 1986; 5: 673-675. 